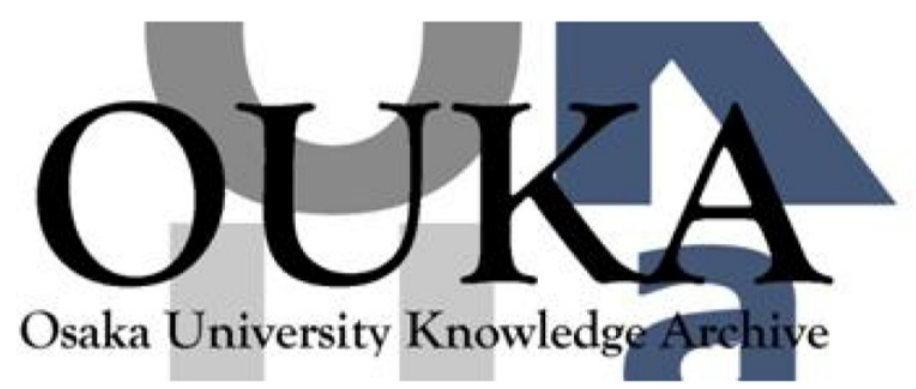

\begin{tabular}{|c|c|}
\hline Title & $\begin{array}{l}\text { Route representation for mobile robot } \\
\text { navigation by omnidirectional route panorama } \\
\text { Fourier transformation }\end{array}$ \\
\hline Author (s) & $\begin{array}{l}\text { Yagi, Yasushi; Fujiwara, Syuji; Yachida, } \\
\text { Masahiko }\end{array}$ \\
\hline Citation & $\begin{array}{l}\text { Proceedings - IEEE International Conference on } \\
\text { Robotics and Automation. } 2 \text { p. } 1250-\text { p. } 1255\end{array}$ \\
\hline Issue Date & $1998-05$ \\
\hline oaire:version & VoR \\
\hline URL & https://hdl. handle. net/11094/14075 \\
\hline rights & $\begin{array}{l}\text { (1998 IEEE. Personal use of this material is } \\
\text { permitted. However, permission to } \\
\text { reprint/republish this material for advertising } \\
\text { or promotional purposes or for creating new } \\
\text { collective works for resale or redistribution } \\
\text { to servers or lists, or to reuse any } \\
\text { copyrighted component of this work in other } \\
\text { works must be obtained from the IEEE.. }\end{array}$ \\
\hline Note & \\
\hline
\end{tabular}

Osaka University Knowledge Archive : OUKA

https://ir. Library. osaka-u. ac. jp/

0saka University 


\title{
Route Representation for Mobile Robot Navigation by Omnidirectional Route Panorama Fourier Transformation
}

\author{
Yasushi YAGI, Syuji FUJIMURA and Masahiko YACHIDA \\ Area of Intelligent Systems, Division of Systems Science \\ Department of Systems and Human Science \\ Graduate School of Engineering Science \\ OSAKA University \\ 1-3 Machikaneyama-cho, Toyonaka, Osaka 560, JAPAN \\ Phone : 06-850-6361 Fax : 06-850-6341 \\ E-mail :y-yagi@sys.es.osaka-u.ac.jp
}

\begin{abstract}
Described here is a route navigation method for a mobile robot with an omnidirectional image sensor. The route is memorized by a series of two dimensional Fourier power spectrums of consecutive omnidirectional images at the horizon while the robot moves to the goal position. While the robot is navigating to the goal point, it is controlled by comparing the principal axis of inertia of its current position with that of the memorized Fourier power spectrum.
\end{abstract}

\section{Introduction}

A geometrical model based method is a typical approach for visual navigation and has been intensively studied $[1,2]$. While the robot is moving around, it takes images and analyzes the images to find obstacles and estimate its own location. With this method, features such as vertical edges extracted from the input image are matched with ones in the image model generated from the environmental model. Generally, the geometrical method is suitable for estimating precise locations of the robot and unknown objects in the environment. However, acquiring accurate geometric information is difficult and expensive for real-time navigation.

On the other hand, memory-based navigation is an another common approach for visual navigation [3-9]. The basic operation is the comparison between present sensorial inputs and previous memorized patterns. It is easy to directly relate the robot action and sensory data without the geometrical model. Zheng's robot memorized the side of scene of a route by a panoramic view while it moved along the route[4]. Matsumoto et al.'s robot memorized the whole front view image at reference points along the route for visual navigation [5]. The correspondence between present input images and previous memorized images were established by using a DP matching method and a correlation method, respectively. However, these methods need a large amount of memory for memorizing the route. Therefore, to reduce memorizing data, several researchers investigated methods for selecting landmarks from memorized scenes $[6,7,8]$, by using disparity information or interesting features such as vertical edges. Ishiguro has proposed another compact representation by expanding it into Fourier series [9]. Each input image is memorized by the coefficients of the low frequency components. His approach is simple and useful for navigation in a real environment. He has shown the result of organized memory in an actual environment; however, the result of autonomous navigation has not been reported yet.

Most of these previous memory-based approaches memorize relations between the apparent features (image) at reference points. Apperant features are usuful information for finding correspondence between the current position and memorized ones and for estimating orientation of the robot. Quantitative information for trajectory following control of the robot was not included in these apparent features at the reference point. Therefore, Usually, thees robots ware controlled by a qualitative method [5]. Apperant featuresat the reference point do not directly represent the robot action against the environment and the spatial relation between the environment and the robot.

In this paper, we propose a memory-based route navigation method for a mobile robot with an omnidirectional image sensor. The route is memorized by a series of two dimensional Fourier power spectrums of consecutive omnidirectional images at the horizon while the robot moves to the goal position. While the robot is navigating the goal point, the robot is controlled by comparing with the pattern of memorized Fourier power spectrum and its principal axis of inertia. The method can directly represent the temporal and spatial relations between the environment and the robot. We performed experiments in an actual indoor environment including a room and a corridor.

\section{Omnidirectional Route Panorama}

A robot moving on a route can see a lot of objects in various directions. A sensor is needed to view the environment around the robot so that it may navigate safely. We have proposed several omnidirectional image sensors such as COPIS, MISS and HyperOmniVision $[10,11,12]$ for robot navigation. These sensors have some important advantages; an omnidirectional view around the robot can be acquired in real-time and all visible vertical edges in the environment appear as line segments radiating 
from the image center, by using a conic or a hyperboloidal mirror. Then, robot navigation becomes simple and reliable by using such omnidirectional image sensors. Omnidirectional image sensors are particularly well suited for visual navigation in a real environment. Fig.1 (a) and (b) are the omnidirectional image sensor COPIS and the example of its image. In this paper, we use the image sequence of the omnidirectional image, called the omnidirectional route panorama, while the robot continuously scans the scenes along the route.

The robot begins to move and takes an image sequence. As shown in Fig.1 (c), each omnidirectional image is

transformed into 2-D polar coordinates $(r, \theta)(r=$ $\sqrt{\left.x^{2}+y^{2}\right)}$, called an omnidirectional panorama image.

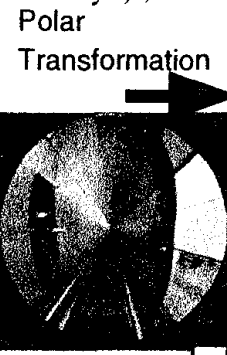

(b)

Omnidirectional Image

Camera center

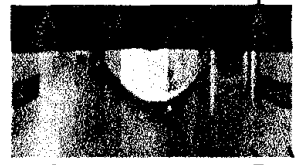

(c) Omnidirectional Panorama Image

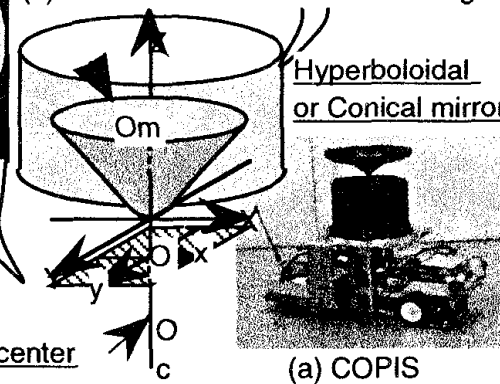

Figure 1 An Omnidirectional Imaging

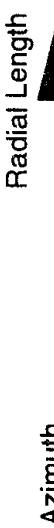

A Sequence of Omnidirectional Panorama Images

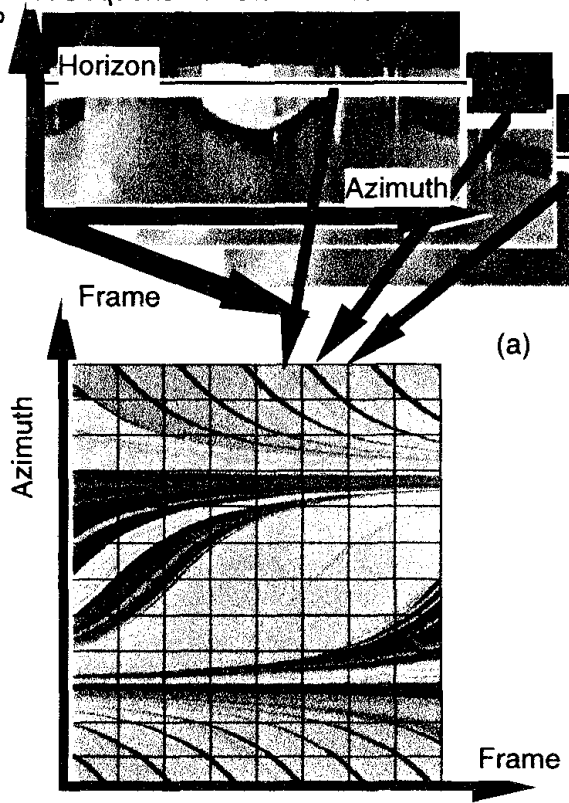

(b)

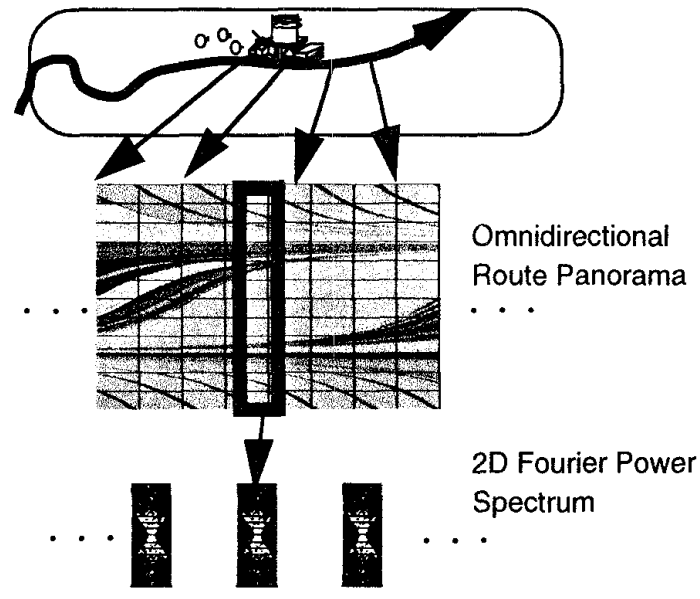
2D Fourier Power Spectrum

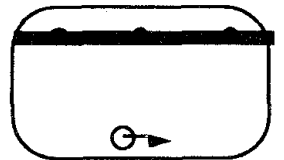

Simple Environment

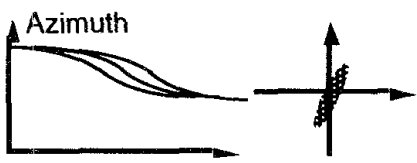

(a) Frame

2D FPS

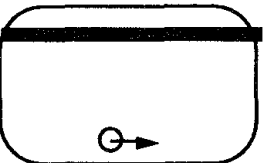

Dense Environment

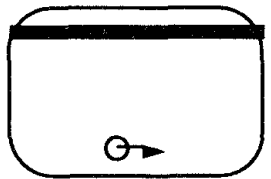

Wide Environment

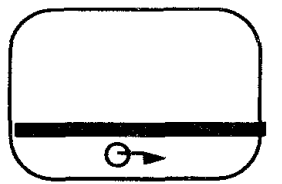

Narrow Environment

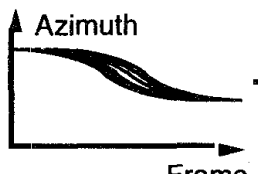

(b)

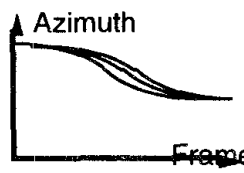

(c)

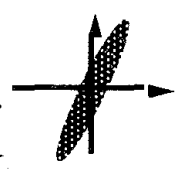

2D FPS
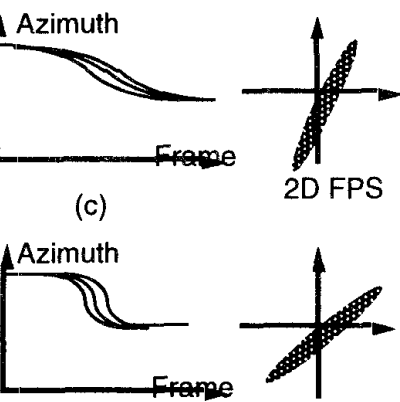

(d)

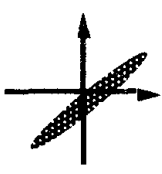

2D FPS
Figure 3 Space Representation by

Figure 4 Relation between Environment and 2D FPS

Points on the horizontal plane, which pass through the virtual center of the lens, appear as a straight line (horizontal line) on the omnidirectional panorama image (drawn by a white line) as shown in Fig.2 (a). An omnidirectional route panorama can be acquired by arranging horizontal lines taken by the robot moving along the route as shown in Fig.2 (b).

\section{Route Representation For Navigation}

An important cue for memory-based navigation is how to represent the relation between the environment and robot behavior. We represent both relations by performing

Figure 2 Omnidirectional Route Panorama 
2-D Fourier transformations on the omnidirectional route panorama.

3.1 Space Representation By Using

2D Fourier Power Spectrum

Suppose that the motion of the robot is linear and constant. As shown in Fig.3, the omnidirectional route panorama in the past certain frames, which is a standard unit of space representation, is transformed to a $2 \mathrm{D}$ Fourier power spectrum (2DFPS). The route is represented by a series of 2DFPSs.

As shown in Fig.4 (a), when the robot moves along a simple environment, patterns on the omnidirectional route panorama are distributed in a lower frequency component in the 2DFPS. On the other hand, if the robot moves in the same size environment, higher frequency components increase in the area where there are dense features as shown in Fig.4 (b). The frequency component of 2DFPSs can represent the complexity of the environment relative to the robot movement and the size of the environment.

The direction of distribution in a 2DFPS is perpendicular to the slope of the tangent of patterns in the Omnidirectional Route Panorama. The pattern indicates the azimuth change of the locus of the feature while the robot is moving. In gerenal, the location of an object can be estimated from the locus of the azimuth of the object by triangulation in the case of constant and linear motion of the robot. As same as such geometrical method, the direction of the distribution in the 2DFPSs includes 3D information.

As shown in Fig. 4 (c), in the case of a long distance between the robot and a wall, patterns on the wall move slowly. The inclination of the distribution in the 2DFPSs increases and the distribution shifts toward a longitudinal axis. On the other hand, as shown in Fig.4 (c), when the robot moves near a wall, the inclination decreases and the distribution shifts toward a transverse axis. Therefore, the inclination of distribution in the 2DFPSs can represent the environment relative to robot location and the robot movement without geometircal reconstruction.

Fig.5 shows comparative results of space representation. Fig.5 (a), (b) and (c) show the omnidirectional route panorama when the robot moved along the middle position of the corridor, when the robot moved along one side of the same corridor and when the robot moved in the computer room respectively. The width of the corridor is approximately $2.4 \mathrm{~m}$. The size of the room is approximately $4 \mathrm{~m}$ by $7 \mathrm{~m}$. Fig. 5 (d) shows the cross-correlation corresponding to the reference 2DFPS. The reference 2DFPS is the first 2DFPS of fig.5 (a). The best matching score of the cross correlation is searched by rotating the 2DFPS around the origin. Even if the robot moves along different routes in the same corridor, values of the cross correlation keep the higher score. On the other hand, when the robot moved in the computer room, the value of the cross correlation was absolutely lower than others. These results mean that 2DFPSs can represent the difference of the environment. Even if the robot moves along different routes in the same corridor, values of the cross correlation keep the higher score. On the other hand, when the robot moved in the computer room, the value of the cross correlation was absolutely lower than others. These results mean that 2DFPSs can represent the difference of the environment.

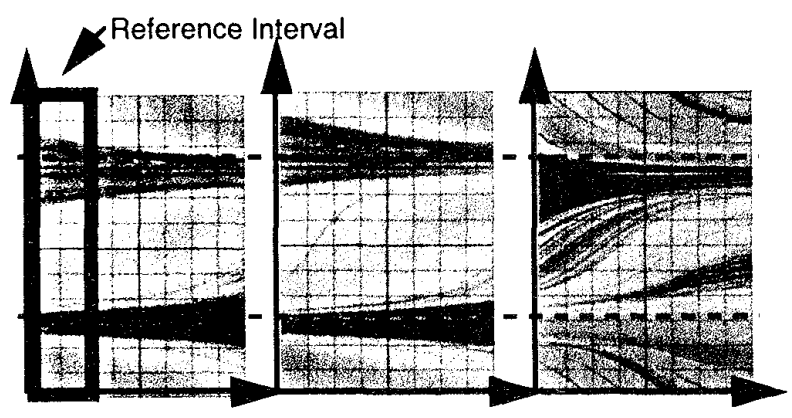

(a) Middle Position (b) One Side

(c) Computer Room

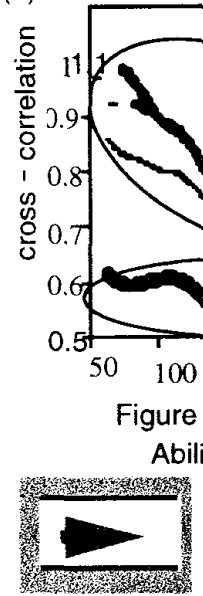

(a) Middle Position (b) One Side

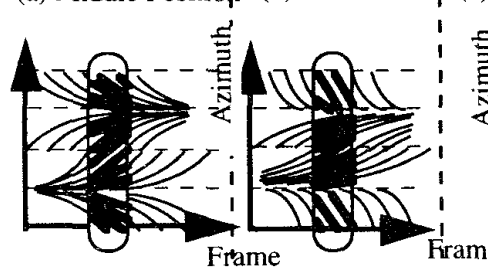

(a) Middle Position

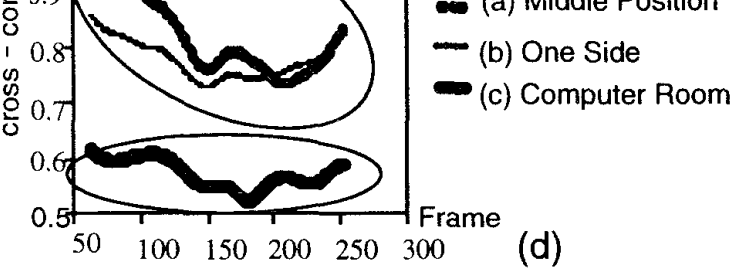

Figure 5 Comparative Experiment of Abilityof Space Representation
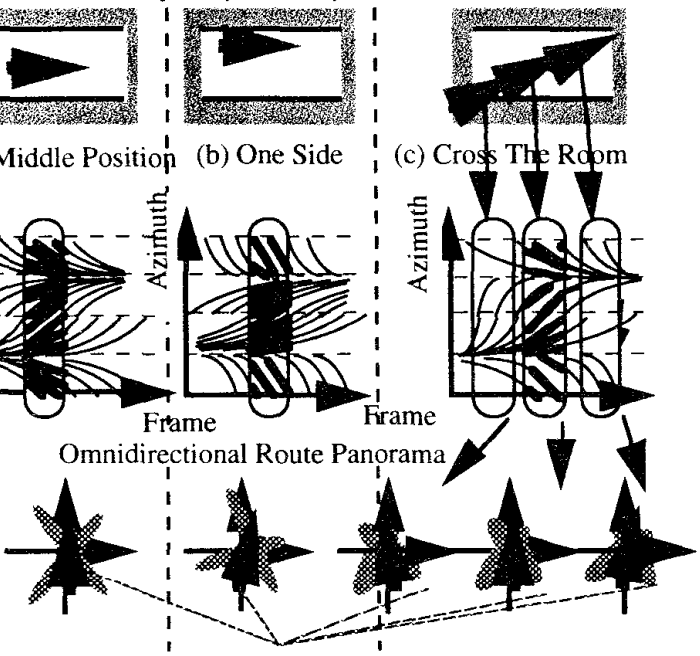

The Principle Axis of The Inertia of The 2D FPS Ordinate axis : Angular Spatial Frequency Transverse axis: Temporal Spatial Frequency

Figure 6 Robot Behavior Representation by Using the PrincipleAxis of Inertia ofThe 2D Fourier Power Spectrum 

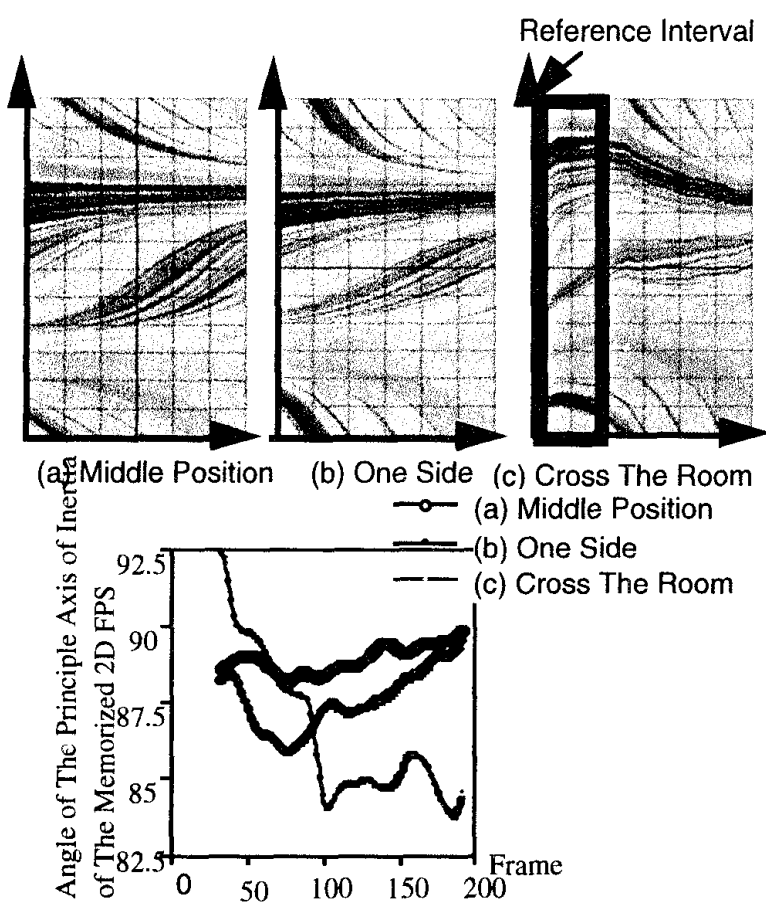

Figure 7 Comparison of The Angle of the principleaxis of inertia of the memorized 2D FPS

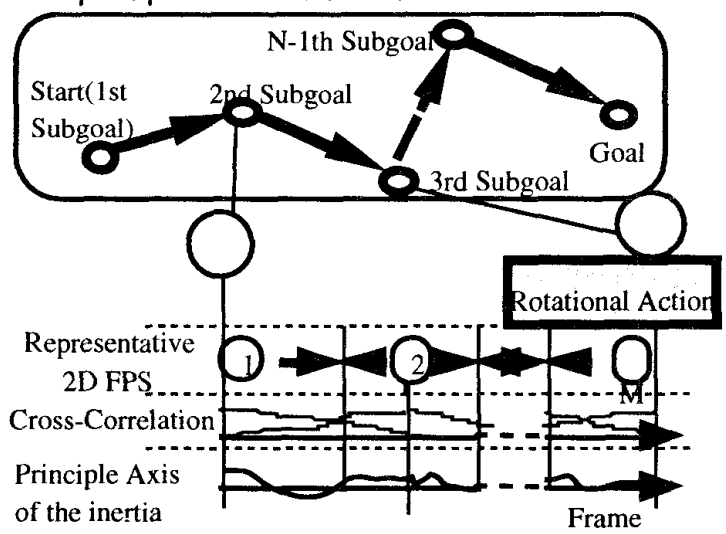

Figure 8 Reference Data for Memory-Based Navigation

In the above explanation, we assume the robot movement is linear and constant. However, the robot, sometime, has to change its direction at an intersection or curved passage in an actual environment. Therefore, we represent a complex route by a series of straight paths as shown in Fig.8. The structural detail of this representation is described in section 4.1.

\subsection{Robot Behavior Representation By Using The Principal Axis of Inertia}

For memory-based navigation, it is necessary to control the robot movement by comparing inputs with memorized patterns. We use the principle axis of inertia of 2DFPS (called PAI) for controlling the robot action.
Consider that the robot moves in a room and both sides of the room have similar texture patterns on the walls. When the robot moves along the middle position of the room, the direction of the PAI is parallel to the room (moving direction), as shown in fig.6 (a). When the robot moves near one wall, the direction of the PAI is tilted in the opposite direction as shown in Fig.6 (b). As shown in Fig.6 (c), even if the robot's moving direction is not parallel to the environment, the direction of the PAI shows similar results.

Fig.7 shows comparative results of the angular change of the PAI against different robot movements in an actual environment. Fig.7 (a) and (b) move along the middle position and to one side of the room, respectively. The size of the room is approximately $4 \mathrm{~m}$ by $7 \mathrm{~m}$. In the case of the middle position, the angular change of PAI is small. On the other hand, a large angular change appears when the robot moves along one side of the room. When the robot moves across the room, the angle of the PAI changes monotonously and its changing direction is opposite to the robot location relative to the room as shown in Fig. 7 (c). Therefore, the locus of angles of the PAI can provide useful information about the robot orientation and position relative to the environment.

\section{Route Navigation Algorithm}

\subsection{Memory Organization}

Following the privous sections, we use the following description for representing the environment and the robot behavior (Fig.8). A route toward the goal position is divided into straight paths and subgoals are set at the joints. The rotational angle of the robot is memorized at each subgoal. To reduce the total amount of iconic memory, each path is represented by a series of representative 2DFPSs which are automatically selected and memorized.

The first 2DFPS is set as the representative 2DFPS. The cross-correlation between the polar transformed representative 2DFPS and the current polar transformed 2DFPS is calculated while the robot is moving. The value of the cross correlation decreases with distance in common environments. If the value of the cross correlation is lower than a certain threshold, we set a flag for a temporal reference point. In the same manner, we extract the next reference point by calculating the cross-correlation between the temporal polar transformed 2DFPS and the current polar transformed 2DFPS. The robot memorizes changes of two cross-correlations while the robot moves: the first is the cross-correlation between the current and the previous polar transformed 2DFPSs, and the other is between the current and the next polar transformed 2DFPSs. Furthermore, the locus of the angle of the principle axis of inertia is memorized. Cross-correlation and the angle of the principle axis of inertia are used for robot control.

\subsection{Autonomous Navigation}

After organizing the iconic memory, the robot can be navigated along the memorized route. The robot is initially parked at a standard position and is driven around 
a room and a corridor of the building via a given route. The rough location of the robot can be identified by comparing the present polar transformed 2DFPS with the polar transformed representative 2DFPS on both sides of the present path. Under constant velocity of the robot, the direction of the robot motion (the steering angle of the robot) is controlled by the common proportional controller defined by the angular difference between the present PAI and the memorized PAI.

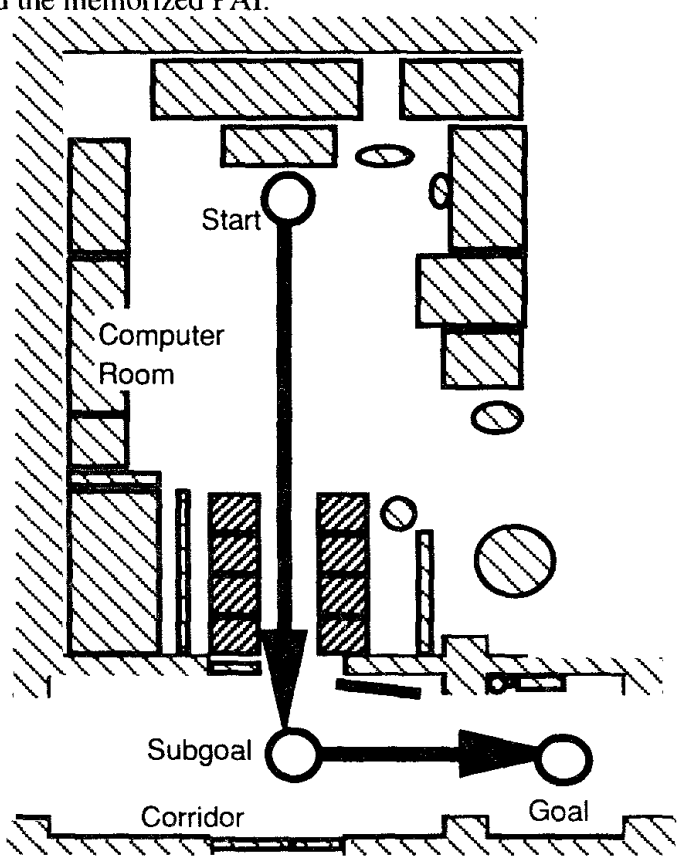

Figure 9 Experimental Environment

\section{Experimental Results}

\subsection{Robot System Configuration}

The system has three essential components; an omnidirectional imaging subsystem, a workstation and a mobile robot. Wireless modems are used for serial communication between the robot and the workstation. The image is transmitted by UHF video transmitter and receiver. The speed of the robot is about $5 \mathrm{~cm} / \mathrm{sec}$ and the size is a diameter of approximately $300 \mathrm{~mm}$.

\subsection{Results of Autonomous Navigation}

Experiments were carried out in our university building. Fig. 9 shows the layout of the experimental environment. The robot was initially parked in our computer room. The operator controlled the mobile robot and moved it toward the door. After passing through the door, the operator turned the robot left in the middle of the corridor. Final the robot was navigated by the operator until the goal position. Totally, the robot moved approximately 10 meters. We did the same experiment twice. The second run was performed on a different day, therefore, the lighting condition was different and a few people were walking in the corridor. The second navigated route was similar but not same as the first one because the robot was controlled by a human operator.

Fig.10(a) and (b) are the omnidirectional route panorama and iconic memorized data including 2DFPSs and cross-correlations as well as the angle of the principle axis of inertia. The threshold for cross-correlation was 0.8 . The window size of a reference route panorama was 360 deg by 32 frames. Upward and downward arrows in Fig. 10 (a) (dotted lines) are respective reference points. We consider that both patterns in Fig. 10 are almost the same, therefore, we consider that reproducible and stable representations can be generated by the proposed method.

Fig. 11 shows the result of autonomous route navigation. Upward arrows are reference points on the memorized route data. Downward arrows are corresponding frames in an omnidirectional route panorama observed during autonomous navigation. Finally, the robot could reach the goal position.

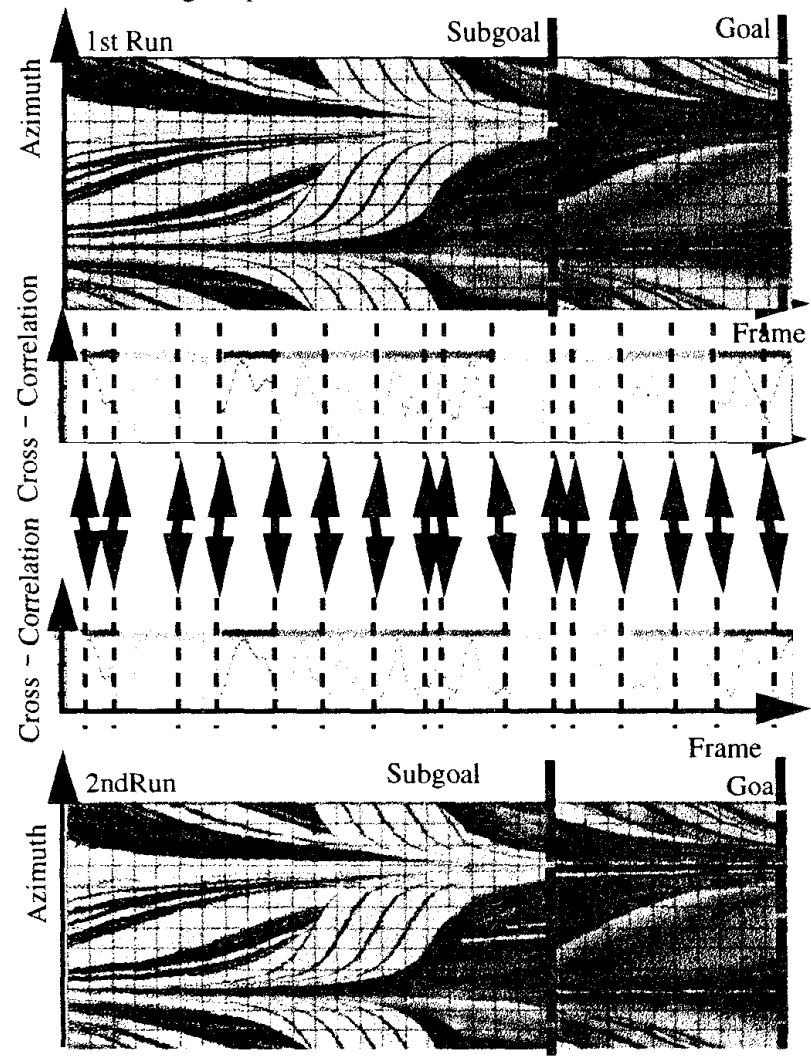

Figure 10-(a) Examples of Memorized Route Data 

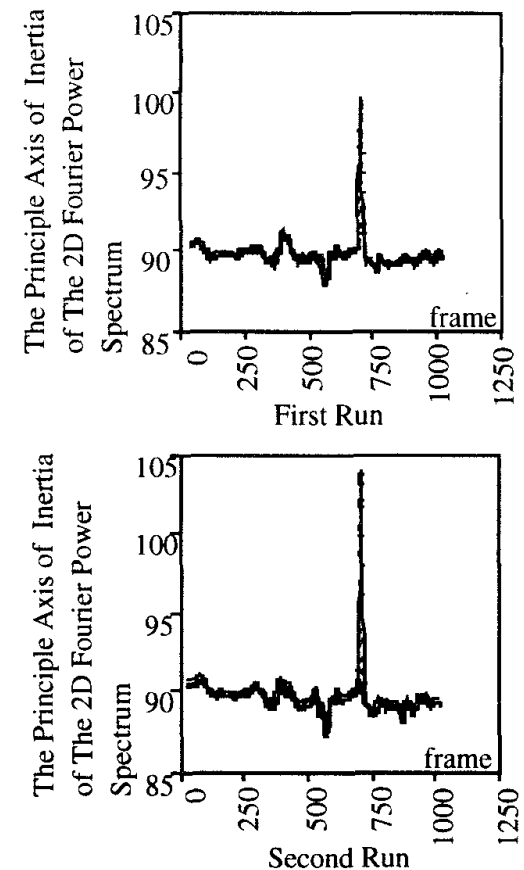

Figure 10 (b) Loci of The Principle Axis of Inertiaof The 2D Fourier Power Spectrum Route Data during autonomous navigation

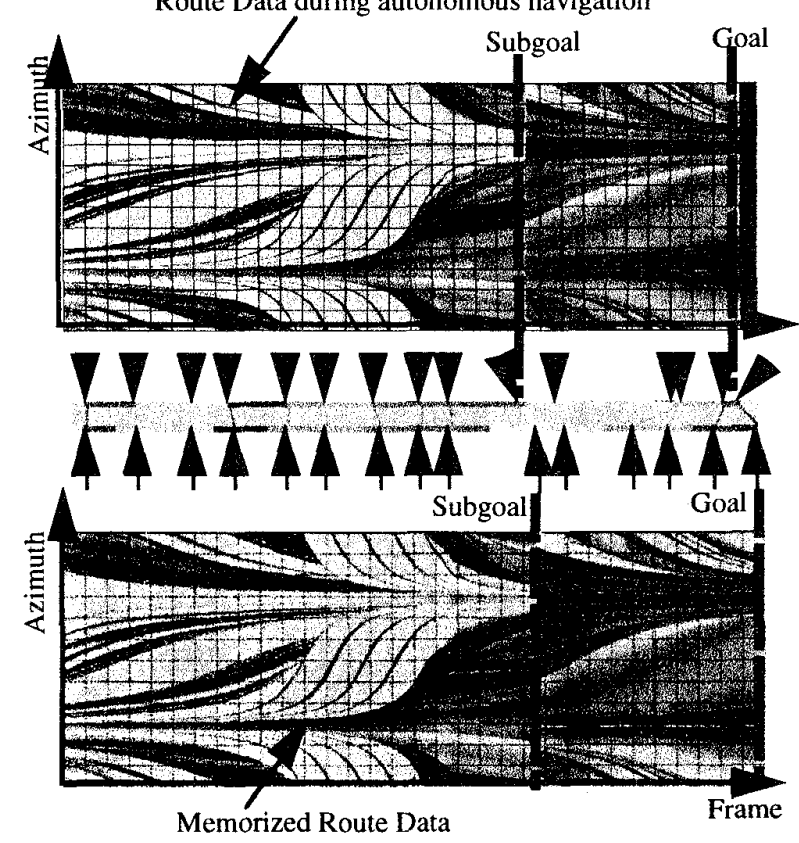

Figure 11 A Result of Autonomous Route Navigation

\section{Conclusions}

In this paper, we proposed a route navigation method for a mobile robot with an omnidirectional image sensor. The route is memorized by a series of two dimensional Fourier power spectrums of consecutive omnidirectional images at horizon while the robot moves to the goal position. The method can directly represent the robot action and the spatial relation between the environment and the robot. We performed experiments in an actual indoor environment including a room and a corridor. A reproducible and stable representation can be generated by the proposed method, and we consider it to be suitable for robot navigation. We are currently trying to navigate a long route in both an indoor and outdoor environment.

Acknowledgement

We thank N.E. Bienson for helpful advice on presentation of this material. This work was partially supported by a Grant-in-Aid for Scientific Research from the Ministry of Education, Science, Sports and Culture, Japanese Government.

\section{References}

[1] M. Yachida, T. Ichinose and S. Tsuji, Model-Guided Monitoring of a Building Environment by a Mobile Robot, 8th IJCAI, Vol.2, pp.1125-1127, (1983)

[2] O.D. Faugeras, E. Le Bras-Mehlman and J. D. Boissonnat ,Representing Stereo Data with the Delaunay Triangulation, Int. J. Artificial Intelligence, 44, pp.41-87, (1990)

[3] $\mathrm{Zhu}, \mathrm{Z} ; \mathrm{Xi}, \mathrm{H} ; \mathrm{Xu}$, Combining rotation-invariance images and neural networks for road scene understanding, ICNN. , vol.3, 1732-1737 (1996)

[4] J. Y. Zheng and S. Tsuji, Panoramic Representation for Route Recognition by a Mobile Robot, IJCV, vol.9, no.1, pp.55-76 (1992)

[5] $\mathrm{Y}, \mathrm{Matsumoto}, \mathrm{M}$. Inaba and $\mathrm{H}$. Inoue, Visual Navigation Based on View-Sequenced Route Representation, J. Robotics Society of Japan, vol.15, no.2, 236-242 (1997) (In Japanese)

[6] T. Ohno, A. Ohya and S. Yuta, Autonomous Navigation for Mobile Robots Referring Pre-recorded Image Sequence, IEEE/RSJ IROS, 2, 672-679 (1996)

[7] J. Hong, X. Tan, B. Pinette and R. Weiss, Imagebased Homing, Proc. IEEE ICRA, pp.620-625, 1991

[8] B. Crespi, C. Furlanello and L. Stringa, Memory Based Navigation, Proc. of the International Jonit Conference on Artificial Intelligence, Robotics and Vision, p. 1654, 1993

[9] H. Ishiguro and S. Tsuji, Image-Based Memory of Environment, IEEE/RSJ IROS 2, 634-639 (1996)

[10] Y. Yagi, S. Kawato, S. Tsuji, Real-Time Omnidirectional Image Sensor (COPIS) for VisionGuided Navigation, IEEE Trans. Robotics and Automation, vol.10, no.1, pp11-22 (1994)

[11] Y. Yagi, H. Okumura and M. Yachida ,Multiple Visual Sensing System for Mobile Robot, Proc. IEEE ICRA,vol.2,1679-1684 (1994)

[12] K. Yamazawa, Y. Yagi and M. Yachida, Omnidirectional Imaging with Hyperboloidal Projection, IEEE/RS.J IROS, 2, pp.1029-1034 (1993). 